\title{
Meal patterns of weaned dairy calves are affected by previous dietary experience and associated with competition surrounding individual feed bins
}

\author{
K. C. Horvath, $\odot$ A. N. Brocious, $\odot$ and E. K. Miller-Cushon* ${ }^{*}$ \\ Department of Animal Sciences, University of Florida, Gainesville 32611
}

\begin{abstract}
The objective of this study was to evaluate how meal patterns of recently weaned and group-housed dairy calves are subject to effects of previous dietary experience and associated with competition for feed. During the preweaning period, Holstein heifer and bull calves were provided diets differing in provision and presentation of forage as follows: (1) pelleted starter only (n $=12$ ), (2) starter and chopped coastal bermudagrass provided in separate buckets $(\mathrm{n}=13),(3)$ starter and chopped coastal bermudagrass mixed together in the same bucket $(\mathrm{n}=15)$, or (4) starter, chopped coastal bermudagrass, and liquid molasses mixed together in 1 bucket $(\mathrm{n}=13)$. At $58 \pm 2$ (mean \pm standard deviation) days of age, following weaning, calves were mingled between treatments and moved into group housing in weekly age-based cohorts $(7 \pm 2$ calves/group; 8 groups total), and all were provided the mixed diet (without molasses). Within group pens, calves were fed individually using the Calan Broadbent feeding system (American Calan Inc.). Calves were monitored for 1 wk following introduction to the group pen. Feed intake was measured daily. Behavioral data, describing feeding times and competition for feed, were recorded continuously for $48 \mathrm{~h}$ beginning after a 5 -d adaptation to the group pen. Calves previously provided starter only had longer, less frequent meals than calves previously provided forage in any form, and they tended to consume less feed compared with those previously provided starter and hay separately, with calves previously provided mixed diets having intermediate intakes. We observed occurrences of displacements at bins, which were followed by replacements and feed stealing on some occasions, where $34 \%$ of calves consumed feed from a bin assigned to another calf on at least 1 occurrence, and $64 \%$ of calves were stolen from at least once.
\end{abstract}

Received June 17, 2021.

Accepted October 6, 2021.

*Corresponding author: emillerc@ufl.edu
Competition at feed bins was not affected by previous dietary treatments, but was associated with meal patterns. Actor displacement rate was negatively associated with meal frequency and duration, but calves that were displaced more often were those that spent more time feeding. These results suggested a possible carryover effect of previous exposure to forage on postweaning meal patterns following a dietary change. Further, we found that competitive behavior varied considerably between individuals, occurring frequently despite feeding calves using individually-assigned feeding bins, and was associated with meal characteristics. These results highlighted the importance of considering both previous dietary experience and social factors when evaluating feeding behavior.

Key words: dairy calf, competition, feeding behavior

\section{INTRODUCTION}

Dairy calves commonly experience housing and dietary transitions surrounding the time of weaning, when previously individually-housed calves may be group-housed for the first time. It has been established that early life experience may shape longer-term patterns of behavior, potentially influencing feed preferences and feeding patterns (reviewed by Miller-Cushon and DeVries, 2015). Further, initial social grouping can be a stressful transition, with evidence of individual variability in adaptation to group housing in a novel environment (Horvath and Miller-Cushon, 2018) and well-established effects of competitive pressure on meal patterns (Crossley et al., 2017; DeVries and von Keyserlingk, 2009).

Previous dietary exposure, in particular, has considerable potential to shape longer-term feeding behavior of dairy calves, and approaches to providing solid feed to preweaning dairy calves vary across the dairy industry. Provision and presentation of forage is variable, and recent research has examined behavioral and performance consequences of providing any access to forage (Khan et al., 2011; Horvath and Miller-Cushon, 2019) 
and the means of presenting it, either separately or as a mixture (Miller-Cushon et al., 2013a,b; Engelking et al., 2020). Previous work has established that forage provision may increase meal frequency and duration (Horvath and Miller-Cushon, 2019) and that forage presentation may additionally influence meal patterns (e.g., tending to increase meal duration when provided as a mixture rather than separate components; MillerCushon et al., 2013a). However, potential longer-term effects of previous exposure to hay on meal patterns remain largely unexplored.

In addition to previous dietary experience, social factors affecting group-housed calves may influence meal patterns. A reduction in meal frequency is often observed to coincide with increased rates of displacement across animals of different ages, including milkfed calves (Miller-Cushon et al., 2014), growing heifers (DeVries and von Keyserlingk, 2009), and adult cows (Crossley et al., 2017). Although meal patterns are quite responsive to competitive pressure, these changes can occur in the absence of consistent effects on total feeding time or feed intake; for example, growing heifers subject to competition for access to their feed increased meal size and duration to compensate for reduced meal frequency such that feed intake was not affected (DeVries and von Keyserlingk, 2009). Competition increases following regrouping (von Keyserlingk et al., 2008), suggesting that competitive pressure may have considerable potential to influence meal patterns upon the initial transition to social grouping in recently weaned calves.

Therefore, the first aim of this study was to characterize effects of previous dietary experience, varying in provision and presentation of forage, on meal patterns following postweaning transition to a common diet and group housing. We hypothesized that previous exposure to hay may have a carryover effect on meal patterns, with calves without previous access to hay potentially having less frequent meals, mirroring previously observed direct effects of hay provision on feeding behavior in the preweaning period. The second aim of this study was to assess the extent of competition within a newly formed social group and associations between competitive pressure and meal patterns. Given some evidence of competition and feed stealing in animals fed using individual intake control systems (Ruuska et al., 2014), we were particularly interested in characterizing the nature of those interactions in calves fed individually in the present study using the Calan Broadbent feeding system (American Calan Inc.). We hypothesized that competitive behavior, including initiated and received displacements at the feed bin, would be associated with meal characteristics and that increased competitive pressure may coincide with reduced meal frequency.

\section{MATERIALS AND METHODS}

\section{Animals and Experimental Design}

Fifty-three Holstein heifer $(\mathrm{n}=45)$ and bull calves $(\mathrm{n}=8)$ were enrolled in the study at the University of Florida Dairy Unit (Hague, FL). Sample size calculations for this study were based on estimated effect sizes of preweaning diet on feeding behavior, as described by Horvath et al. (2021). All study procedures were reviewed and approved by the University of Florida Animal Care and Use Committee (protocol \#201609416). During the preweaning period, dairy calves were housed individually and randomly assigned at birth to receive only starter (STR; n = 12), starter and chopped hay (2.5-cm chop length; coastal bermudagrass) in separate buckets ( $\mathbf{S E P} ; \mathrm{n}=13$ ), starter and chopped hay mixed together in the same bucket, at a rate of $80 \%$ starter and $20 \%$ hay ( $\mathbf{M X} ; \mathrm{n}=15)$, or starter, chopped hay, and liquid molasses mixed together into 1 bucket, with molasses added at a rate of $10 \%$ (as-is basis) to the same diet provided to the MX treatment (MXL; $\mathrm{n}=$ 13) for the first $8 \mathrm{wk}$ of life. Diet chemical composition is reported in Table 1. Preweaning data from some of the animals enrolled in the present study (calves from SEP, MX, and MXL treatments, but not STR treatment) was previously reported by Horvath et al. (2021), where we describe preweaning calf management in more detail, alongside results describing calf feed intake and diet selection behavior during the preweaning period. Preweaning data from calves from the STR treatment was not reported by Horvath et al. (2021), but these calves were enrolled concurrently and managed identically. As described in more detail by Horvath et al. (2021), calves were weaned over a 10-d period beginning at $42 \pm 3 \mathrm{~d}$ (mean \pm SD) of age.

In the present study, data collection began upon group housing following weaning. At $58 \pm 2$ (mean \pm SD) d of age, pending veterinary approval following a clinical health exam, calves were mingled between treatments and moved into group housing, in weekly age-based cohorts ( $7 \pm 2$ calves/group; range $4-10 ; 8$ groups total). One group was formed per week, with group size varying dependent on the number of calves born within the same week. The composition of the group in terms of calves previously exposed to different dietary treatments also varied, as calves were assigned randomly at birth to different dietary treatments (as described by Horvath et al., 2021), but each group consisted of at least 1 calf from each treatment. The group pen $(6.7 \times 13.0 \mathrm{~m})$ was sand-bedded and located under an open-sided building to provide shelter from rain. The diet upon moving into group housing was switched to the MX diet (mixture of starter and hay; Table 1). 
Table 1. Chemical composition of feed components and mixed rations (mean \pm SD; DM basis) provided during the preweaning period ${ }^{1}$

\begin{tabular}{lcccc}
\hline Chemical composition $^{2}$ & Hay $^{3}$ & Starter $^{4}$ & Mixed ration & Mix + LF $^{6}$ \\
\hline DM, \% & $88.7 \pm 0.4$ & $92.1 \pm 0.01$ & $91.4 \pm 0.09$ & $88.9 \pm 0.10$ \\
NDF, \% of DM & $73.8 \pm 4.0$ & $26.6 \pm 2.9$ & $33.0 \pm 2.4$ & $31.2 \pm 1.8$ \\
ADF, \% of DM & $44.3 \pm 1.3$ & $14.4 \pm 1.2$ & $19.1 \pm 1.5$ & $18.1 \pm 1.1$ \\
NFC, \% of DM & $10.0 \pm 2.6$ & $42.1 \pm 2.5$ & $37.9 \pm 1.6$ & $40.4 \pm 1.4$ \\
CP, \% of DM & $9.6 \pm 1.1$ & $22.8 \pm 0.2$ & $21.1 \pm 0.94$ & $20.8 \pm 0.71$ \\
Starch, \% of DM & $5.7 \pm 0.83$ & $26.4 \pm 1.2$ & $20.9 \pm 1.4$ & $21.0 \pm 1.3$ \\
NDFICP, \% of DM & $2.2 \pm 0.09$ & $2.2 \pm 0.18$ & $2.6 \pm 0.16$ & $2.5 \pm 0.15$ \\
Ether extract, \% of DM & $1.2 \pm 0.19$ & $2.8 \pm 0.09$ & $2.7 \pm 0.18$ & $2.8 \pm 0.17$ \\
Ash, \% of DM & $7.7 \pm 0.50$ & $8.0 \pm 0.17$ & $8.2 \pm 0.14$ & $8.6 \pm 0.13$ \\
TDN, \% of DM & $49.9 \pm 1.3$ & $74.3 \pm 1.1$ & $71.7 \pm 1.1$ & $72.4 \pm 0.88$ \\
ME, Mcal/kg of DM & $1.8 \pm 0.05$ & $2.7 \pm 0.04$ & $2.6 \pm 0.04$ & $2.6 \pm 0.05$ \\
\hline
\end{tabular}

${ }^{1}$ Table adapted from Horvath et al. (2021), where preweaning exposure to different dietary treatments is described and reported here for the reader's convenience. Methodology for feed sampling and analysis are described by Horvath et al. (2021).

${ }^{2}$ Values were obtained from chemical analysis of feed samples. NFC $\%=100 \%-[\mathrm{CP} \%+(\mathrm{NDF} \%-\mathrm{NDFICP}$ $\%)+$ ether extract\% + ash\%]. ME $=$ TDN $\times 0.04409 \times 0.82$ (calculated according to NRC, 2001 equations). NDFICP $=$ NDF-insoluble CP.

${ }^{3}$ Second-cut coastal bermudagrass hay (chopped $<2.5 \mathrm{~cm}$ )

${ }^{4}$ Pelleted calf starter; Ampli-Calf Starter Warm Weather, Purina Animal Nutrition LLC.

${ }^{5}$ Contained, on a DM basis: $80 \%$ starter and $20 \%$ hay.

${ }^{6}$ Contained, on a \% of DM: $75 \%$ starter, $18 \%$ hay, and 7.4\% liquid molasses additive (the same 80:20 mixture of starter and hay was mixed with liquid molasses at $10 \%$ on as-is basis). The liquid molasses additive was cane molasses diluted with water to reach $61 \%$ DM, with a low level of sulfuric acid added to reduce stickiness (supplied by Quality Liquid Feeds).

Therefore, the first day in the group pen coincided with the first day of exposure to the mixed diet (for calves not previously assigned the MX treatment during the preweaning period) and the first day of exposure to hay for all calves but for those on the STR treatment. Within group pens, calves were fed individually using the Calan Broadbent feeding system (see Figure 1) to restrict access to each calf's individual feed bucket. The diet was provided $1 \times / \mathrm{d}$ at $1000 \mathrm{~h}$, with feed offered ad libitum, targeting $20 \%$ orts. The group pen was equipped with 18 Calan gates such that stocking density was consistently below capacity for all groups. Calves were randomly assigned to adjacent gates.

Calves were trained to open their assigned Calan gate during 3 training sessions per day (at $0900 \mathrm{~h}$, which preceded morning feeding, $1300 \mathrm{~h}$, and $1600 \mathrm{~h}$ ) for the first $5 \mathrm{~d}$ after grouping. During the first training sessions, each calf was individually led to their gate, and the gate was manually opened by study personnel so the calf could eat. After beginning to eat, the gate was allowed to gently close to rest on their neck, as it would when the calf accessed feed independently. Once calves were comfortable continuing to eat with the gate resting on their neck, the gate was then unlocked but not manually opened by study personnel, such that the calf had to push it open to begin to eat. Once calves accessed their feed bin independently by pushing it open when unlocked, the bin was then locked such that the calf had to lower their head to unlock the gate before opening it (so that the gate would register the transponder attached to the calf's collar). During each training session, personnel only intervened if the calf failed to open the gate independently, by first leading the calf to their assigned gate and then opening it if the calf failed to do so independently. Intervention during training sessions ceased when the calf was consuming feed independently throughout the day without assistance. The majority of calves were able to successfully open and use the gates within 3 to $5 \mathrm{~d}$ without assistance (10.8 \pm 2.4 assists; mean \pm SD across all calves; with 2 calves each requiring 1 final assist on the morning of $\mathrm{d}$ 6 after grouping).

Calves remained in their postweaning grouping for $10 \mathrm{~d}$. There was a 2-d overlap between groups (with a new weekly cohort of calves entering on $\mathrm{d} 8$, and the previous group of calves leaving $2 \mathrm{~d}$ later) that did not coincide with the focal observation days, described below.

\section{Data Collection}

Feed intake was measured daily by weighing the amount of feed offered and removing and weighing the refused feed immediately before offering fresh feed. To determine DMI, fresh feed (pooled sample) was sampled daily, and orts were sampled from individual 

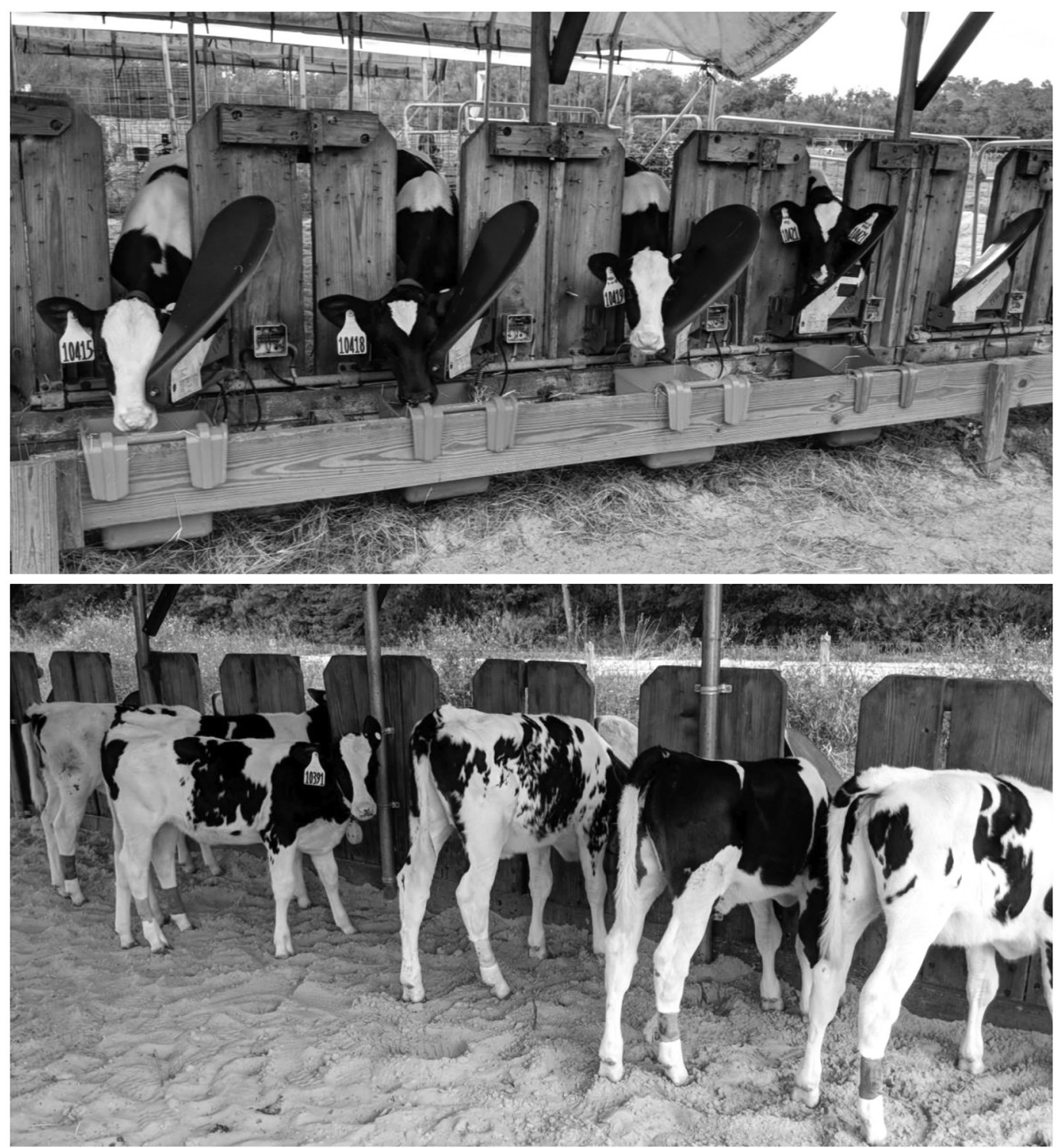

Figure 1. Configuration of feeding spaces in the group pen, showing calves feeding at assigned Calan gates (American Calan Inc.) from the pen exterior (top image) and interior (bottom image).

calves daily and oven-dried at $55^{\circ} \mathrm{C}$ for $48 \mathrm{~h}$. During the period of training each calf to open their Calan gate, as described above, we recorded the number of assists required before the calf accessed their feed independently, as a measure of ability to learn this task. These data were examined in relation to competition and feed stealing surrounding Calan gates, as described in detail below.

To observe feeding patterns and behavior surrounding Calan gates, a video camera (FI9900P; Foscam) was mounted to provide a view of the whole group pen. Behavioral data were continuously recorded for 2 consecutive 24 -h periods (d 6 and 7 following introduction to the group pen) using Behavioral Observation Research Interactive Software (BORIS v. 9.4; Friard and Gamba, 2016). This observation period was selected at the end of the week to maximize the period of learning to access the Calan gates before introducing the next weekly cohort of calves to the group pen, as described above. We characterized the frequency and duration of feeder visits, defined when the calf's head was through the open Calan gate. Observation of social interactions surrounding Calan gates supported the occurrence of displacements at feed bins, as well as instances of "stealing" (with an actor replacing the recipient calf after displacement and continuing to feed from that other 
calf's bin). We characterized the frequency of these displacements (defined as an actor contacting another calf, resulting in their removal from the feeding space) and occurrences of replacing another calf following displacement and continuing to feed from that calf's bin (stealing; defined as the calf having their head through the Calan gate assigned to another calf, with identity of actor and recipient noted). These outcomes describing feeding at the incorrect bin were summarized by actor (duration of stealing from another bin) and recipient (duration of being stolen from by another calf). Two observers characterized these behaviors from video (interobserver reliability was calculated for 2 calves during $1 \mathrm{~d}$ of video collection, with Cohen's kappa $\geq 0.95$, as calculated in BORIS).

\section{Behavioral Data Analysis}

For descriptive purposes, we initially visualized competitive relationships between calves using social network analysis (using Gephi, v 0.9.2; Bastian et al., 2009). We constructed directed networks with edges (i.e., ties between calves) weighted by duration of stealing summed across both observation days. Daily duration of stealing and being stolen from were highly skewed (with 0 values for many calves), and so the occurrences of stealing or being stolen from were converted to binary outcomes for each calf on each observation day $(0=$ no occurrence; $1=1$ or more occurrence during the focal day) for further analysis as described below. Displacements (total, regardless of whether they preceded stealing from the incorrect bin) were summarized as rates for both actor (number of displacements of another calf per hour of feeding time) and recipient (number of times displaced by another calf per hour of feeding time).

Observations of feeder visits from video suggested that calves often opened and closed their Calan gate multiple times in quick succession, rather than opening it 1 time and feeding for a prolonged period. Therefore, we conducted a bout analysis to group visits to the Calan gate (i.e., times when the gate was opened) into longer meals, using methodology described previously (Horvath and Miller-Cushon, 2019). For this analysis, we fit 2 normal distributions (using the $\mathrm{R}$ package mixdist; Macdonald and $\mathrm{Du}, 2018)$, to the $\log 10$ transformed intervals between feeder visits (defined as head through Calan gate). During initial screening of the data, interval distributions for separate treatments were determined to be visually similar, and thus these data were pooled across all calves for analysis to improve model fit (as described by Horvath and MillerCushon, 2019). The meal criterion was determined to be $13.2 \mathrm{~min}$, and this was used to calculate daily meal frequency and duration for individual calves.

For each calf, we summarized daily feeding time, frequency of visits to the feed bin (i.e., number of times they opened the gate to the feed bin), meals (as defined per the bout analysis described above), and duration of feeding time per visit to the feed bin and per meal. We additionally calculated the ratio of visits to the feed bin per meal (reflecting the frequency with which a calf opened and closed the Calan gate within the same meal), and the percentage of meal time spent feeding (reflective of how continuously a calf fed within their meal, as opposed to taking more frequent or longer nonfeeding pauses). These feeding behavior outcomes were summarized across all occurrences of feeding, either from the focal calf's assigned bin or during instances of feeding from another calf's bin.

\section{Statistical Analysis}

Video was missing for a total of 4 calves ( 1 calf from each treatment) for $1 \mathrm{~d}$ of the observation period due to camera failure; therefore behavior of these calves was recorded from $1 \mathrm{~d}$ only. Effects of previous dietary treatment on meal characteristics, DMI, and ADG were analyzed in a general linear mixed model using the MIXED procedure of SAS (version 9.4; SAS Institute Inc.). All data were screened for normality using the UNIVARIATE procedure within SAS before analysis, model residuals were screened for normality, and DMI was square-root transformed to meet assumptions of normality. The model included the fixed effects of treatment and the binary outcomes of feeding from the wrong bin (stealing) or having another calf feed from the focal calf's bin (stolen from; $0=$ no occurrence; $1=1$ or more occurrence during the focal day), group (describing weekly cohort in which the calf was housed in the group pen) as a random effect, and day as a repeated measure, fitted with the compound symmetry covariance structure (except for ADG, where we had only 1 value for the observation period) according to Schwarz's Bayesian information criterion. For the analysis of DMI, data points were not excluded on the basis of observed feed stealing, as the majority of calves either stole feed or were stolen from, but these effects were included in the model, as described above. Although we had no hypotheses regarding an interactive effect of previous dietary treatment and competition on meal characteristics, we examined potential effects of these interaction terms (between treatment and occurrence of stealing, and treatment and occurrence of being stolen from); they were nonsignificant in all cases, and thus excluded from the final models. 
Before the analysis described above, we also evaluated potential effects of previous dietary treatment on competition surrounding feeding. Actor and recipient displacement rates were analyzed in a similar model as described above, without the inclusion of the binary outcomes describing stealing and being stolen from. Displacement rates were square-root transformed to meet model assumptions of normality. Binary outcomes of stealing and being stolen from were analyzed using a linear mixed model with a binary distribution (Proc GLIMMIX), with treatment as a fixed effect and day included as a repeated measure with an unstructured covariance structure. To examine a posthoc hypothesis that displacement or feed stealing may relate to individual ability to access the Calan gate, we examined the relationship between the number of assists required to feed independently and occurrences of calves stealing or being stolen from. The number of required assists was modeled using a negative binomial distribution (Proc GLIMMIX), with the binary categorization of calves based on any occurrence of stealing included as a fixed effect and group included as a random effect. For this analysis, we considered only the first day of observation because categorization based on occurrence of stealing or being stolen from was not consistent across observation days for all calves.

There was considerable individual variability in the rate of displacements at the Calan gate; therefore, we further explored the relationship between displacement frequency (as actor and as recipient) and meal characteristics using linear regression to assess associations between competitive pressure and feeding behavior. Across both focal days, we averaged displacement rate (per hour of feeding time, as actor and receiver), as well as meal characteristic outcomes. Unconditional associations between displacement rates and meal characteristics were analyzed using the ROBUSTREG procedure of SAS. All values reported are least squares means. Significance was declared at $P<0.05$, and trends were reported if $0.05 \leq P \leq 0.10$.

\section{RESULTS}

\section{Characterization of Competition Surrounding Calan Gates}

Across the 2 focal observation days, we observed occurrences of calves displacing one another, which sometimes preceded accessing the feed bin assigned to the other calf. Over the 2 focal observation days, $91 \%$ of calves (48 of 53) displaced another calf at least once. For those calves, the rate of displacing another calf was 3.4 times/h of feeding (median; 95\% CI: 0.46, 21.9).
Over the 2 focal observation days, $92 \%$ of calves (49 of 53 ) were displaced by another calf at least once. Those calves were displaced at a rate of 3.0 times/h of feeding (median; 95\% CI: 0.94, 8.6).

Occurrences where calves replaced a displaced calf (stealing feed from the incorrect bin) were visualized using weighted, directed social networks for descriptive purposes (shown for a subset of pens in Figure 2 ), illustrating considerable individual variability. We found that $34 \%$ of calves (15 of 53) consumed feed from the feed bin assigned to a displaced calf (stealing) on at least 1 occurrence. For those calves that stole feed, $20.4 \%$ (median; $95 \%$ CI: $0.49,87.0$ ) of their total daily feeding time was spent feeding from the incorrect bin, and $24.7 \%$ (median; 95\% CI: 5.6, 85.7) of their daily visits to a feeding bin were to an incorrect bin, following displacement of another calf. We found that $64 \%$ of calves (34 of 53) were replaced at their feed bin by a calf that had displaced them (stolen from) on at least one occurrence. For those calves that were stolen from, 13.5\% (median; 95\% CI: 1.4, 33.8) of the feeding time at their assigned bin and 8.3\% (median: 95\% CI: 2.9, 46.2) of the visits to their assigned bin were occupied by the incorrect calf. There were 7 calves $(13 \%)$ that both stole from another calf's bin and were stolen from on at least 1 of the 2 focal days. There were 9 calves (17\%) that neither stole nor were stolen from across both focal days.

We found no evidence to support an association between ability to learn to open the Calan gate and occurrence of stealing: the number of required assists to feed independently [overall mean 10.8 assists (95\% CI: 9.6, 12.6)] did not differ between calves that stole at least once and those that did not $\left(F_{1,44}=0.00 ; P=\right.$ $0.95)$.

\section{Effects of Previous Dietary Experience on Feeding Behavior}

Following transition to the common mixed diet, DMI tended to differ between treatments $\left(F_{3,46}=2.27 ; P=\right.$ 0.093; Figure 3 ), with calves previously provided starter and hay separately tending to have greater intake than calves previously provided starter only $(P=0.070)$ and calves provided either mixed diets having intermediate intakes (which did not differ from other treatments; $P$ $>0.22)$. Dry matter intake increased over time $\left(F_{6,320}\right.$ $=40.4 ; P<0.001)$ with no treatment by day interaction $\left(F_{18,320}=1.28 ; P=0.20\right)$. These results must be interpreted with caution given evidence of calves feeding from incorrect bins, although there was no effect of previous dietary treatment on displacement rate or the occurrence of stealing or being stolen from (Table 2 ). Although we did not exclude data on the basis of 

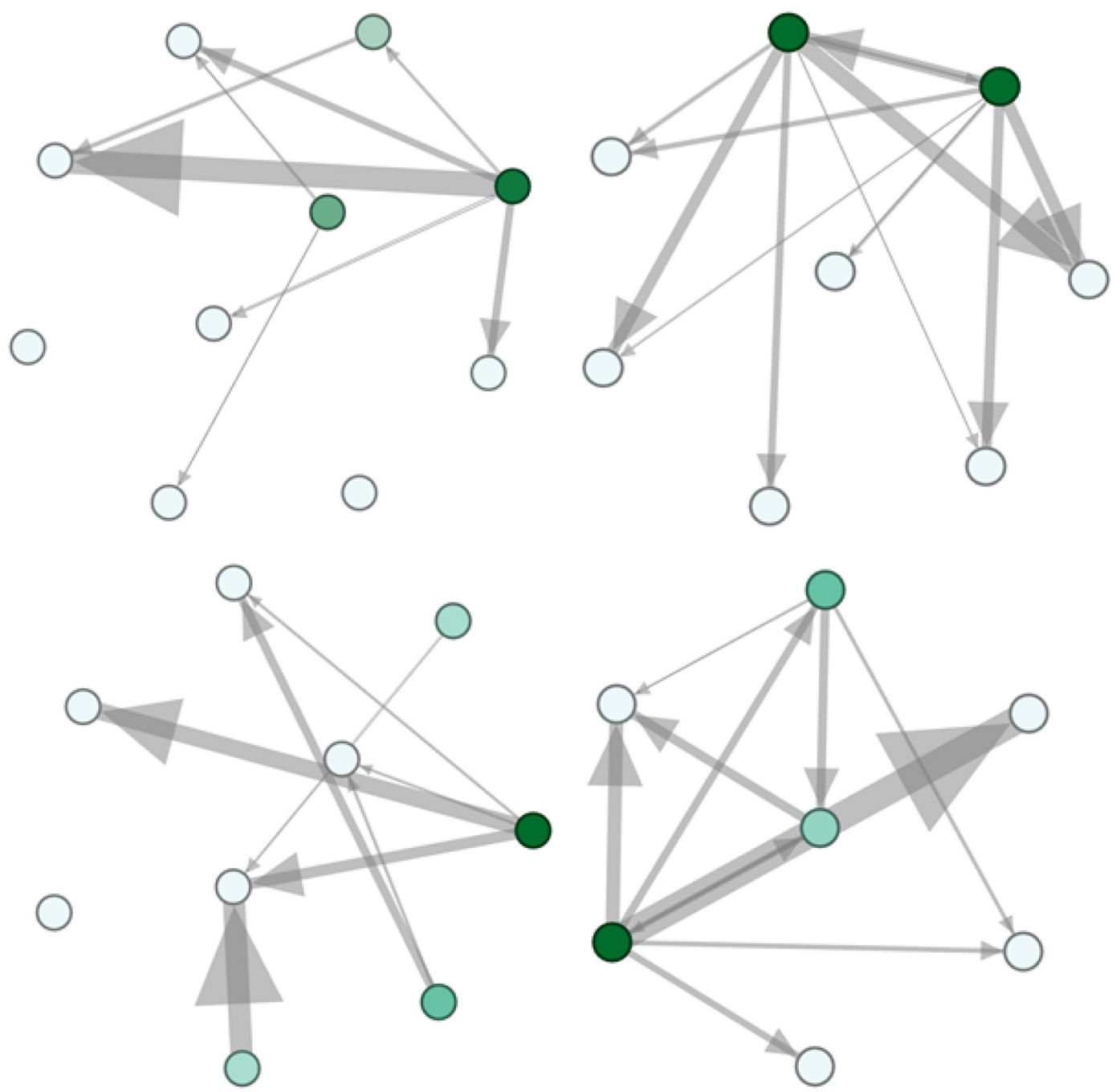

Figure 2. Visualization of the weighted, directed social competition networks, shown for a subset of 4 group pens. Arrow width is scaled to reflect the duration of feeding from another calf's assigned bin ("stealing," observed across 2 observation days), with wider arrows indicating a greater duration of stealing. The node (circles represent individual calves) color gradients are based on out-degree centrality, a measure describing the number of connections the calf has. Darker colors indicate greater out-degree centrality values, meaning that they had more stealing directed toward other calves. Networks were obtained (with out-degree centrality calculated) using Gephi (v. 0.9.2, Bastian et al., 2009) software, shown using a Fruchterman Reingold layout.

stealing, as it affected the majority of the calves, the binary outcomes of stealing and being stolen from were also included in this model; being stolen from predictably coincided with increased DMI from the focal calf's assigned feed bin (1.49 vs. $1.25 \mathrm{~kg} / \mathrm{d}$; any occurrence of being stolen from vs. no occurrence; $\mathrm{SE}=0.09 ; P$ $=0.0095)$, and stealing coincided with decreased DMI at the assigned bin $(1.13$ vs. $1.61 \mathrm{~kg} / \mathrm{d} ; \mathrm{SE}=0.12 ; P$ $<0.001)$. Previous dietary treatment had no effect on ADG during the postgrouping period after transition to the common mixed diet $(0.52$ vs. 0.53 vs. 0.54 vs. 0.43 $\mathrm{kg} / \mathrm{d}$; STR vs. SEP vs. MX vs. MXL; SE $=0.062 ; F_{3,43}$ $=0.70 ; P=0.56)$.
We saw effects of previous dietary treatments on meal characteristics (based on all instances of feeding from any bin) upon transition to the common mixed diet and social grouping (Table 2). Calves previously provided only starter had longer, less frequent feeder visits and meals than calves previously provided forage in any form, and spent a greater percentage of meal time (based on bout analysis) consuming feed. This suggested that they fed more continuously, taking fewer or shorter pauses within their meal. We found no effects of previous dietary treatment on competitive behavior (displacement rates or occurrence of stealing or being stolen from; Table 2). 


\section{Associations Between Competition and Meal Patterns}

The occurrence of feeding from the wrong bin at any time on the focal day (stealing) was associated with reduced visits to the feed bin (summarized across behavior directed toward any feed bin; 19.7 vs. 30.7 gate opens/day; any occurrence of stealing from another calf's bin vs. no occurrence; $\mathrm{SE}=3.3, F_{1,43}=8.81$; $P=0.005$ ). However, occurrence of stealing was not associated with meal frequency (based on bout analysis; $F_{1.43}=0.04 ; P=0.84$ ). Correspondingly, stealing was associated with a lower ratio of feed bin visits per meal (2.2 vs. 4.2 gate opens/meal; any occurrence of stealing vs. no occurrence; $\mathrm{SE}=0.5 ; F_{1,43}=13.7 ; P<$ 0.001). Any occurrence of stealing was also associated with shorter meal durations (16.4 vs. $21.7 \mathrm{~min} / \mathrm{meal}$; $\left.F_{1,43}=6.05, P=0.018\right)$ and a tendency for reduced daily total meal time (122.8 vs. $155.8 \mathrm{~min} / \mathrm{d} ; F_{1.43}=$ $3.12, P=0.084)$, whereas daily duration of feeding time was not associated with stealing $\left(F_{1,43}=0.98 ; P=\right.$ 0.33). Correspondingly, stealing feed from other bins corresponded with a greater percentage of meal time engaged in feeding versus nonfeeding pauses within the meal ( 82.8 vs. $72.8 \%$; $\mathrm{SE}=2.8 ; F=9.61 ; P=0.0034$ ), suggesting more continuous feeding. Calves that were stolen from (following displacement by another calf) had longer feeding times (109.4 vs. $92.0 \mathrm{~min} / \mathrm{d}$; $\mathrm{SE}=$

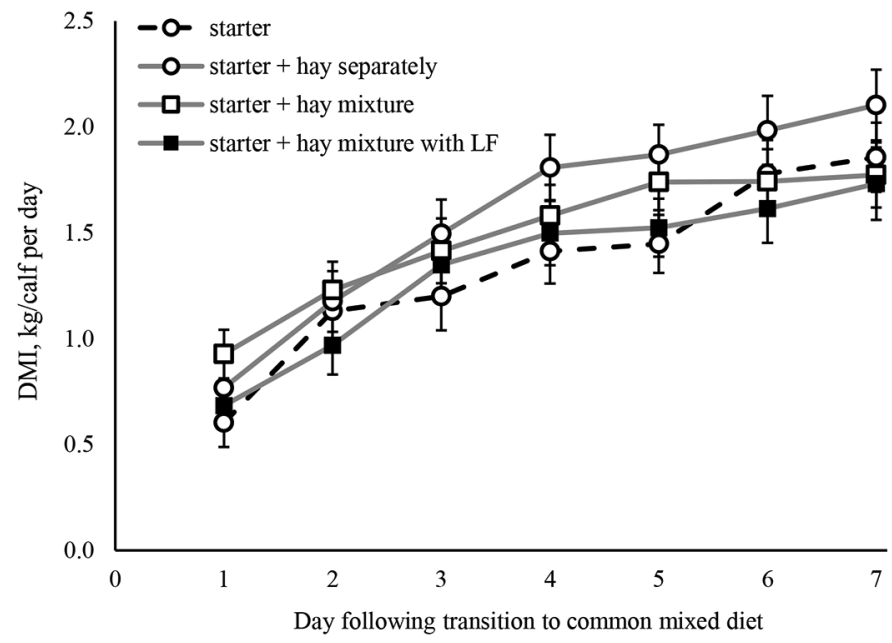

Figure 3. Dry matter intake of calves during the first week following grouping and transition to a common mixed ration $(80 \%$ starter and $20 \%$ hay). Calves were previously provided diets varying in provision and presentation of hay as follows: starter only ( $\mathrm{n}=12$ calves), starter and hay separately $(\mathrm{n}=13)$, mixed diet of $80 \%$ starter and $20 \%$ hay $(\mathrm{n}=15)$, and the mixed diet containing molasses-based liquid feed $(\mathrm{LF})$ at $10 \%(\mathrm{n}=13)$. Solid lines indicate provision of hay, square markers indicate the diet was provided as a mixture, and the addition of LF is indicated with filled markers. Values are back-transformed LSM, with error bars representing $95 \%$ CI. $\left.7.4 ; F_{1,43}=4.2 ; P=0.047\right)$, as well as longer, more frequent meals $\left(8.8\right.$ vs. 7.3 meals $/ \mathrm{d} ; \mathrm{SE}=0.7 ; F_{1.43}=$ 4.13; $P=0.048)$. Otherwise, other meal characteristics did not differ between calves stolen from at least once and those not stolen from $(P>0.3)$.

Further investigation of the relationship between the extent of competitive pressure and feeding behavior revealed associations between the rate of being displaced and displacing another calf and meal characteristics (Figure 4). The recipient displacement rate (number of times the calf was displaced by another calf per hour of feeding time; $3.5 \pm 3.4$ events/h of feeding; mean \pm SD) was positively associated with meal frequency (estimate $=0.53 ; \mathrm{SE}=0.19 ; P=0.0049 ;$ Figure 4a), daily total duration of meal time (estimate $=11.8 ; \mathrm{SE}$ $=3.1 ; P<0.001 ;$ Figure $4 \mathrm{~b}$ ), and daily frequency of feeder visits (i.e., opening the gate; estimate $=3.1$; SE $=0.63 ; P<0.001$; Figure $4 \mathrm{c}$ ), and was negatively associated with feeding time, as a \% of meal time (estimate $=-2.1 ; \mathrm{SE}=0.53 ; P<0.001 ;$ Figure $4 \mathrm{~d})$.

In contrast, the actor displacement rate (number of times the calf displaced another calf per hour of feeding time; $5.3 \pm 7.1$ events/h of feeding; mean $\pm \mathrm{SD}$ ) was negatively associated with meal frequency (estimate $=$ -0.19 ; $\mathrm{SE}=0.094 ; P=0.042$; Figure $4 \mathrm{e}$ ), daily total duration of meal time (estimate $=-5.2 ; \mathrm{SE}=1.32$; $P<0.001 ;$ Figure 4f), and daily frequency of feeder visits (estimate $=-0.98 ; \mathrm{SE}=0.34 ; P=0.0045 ;$ Figure $4 \mathrm{~g}$ ), and tended to be positively associated with feeding time, as a $\%$ of meal time (estimate $=0.60 ; \mathrm{SE}=0.32$; $P=0.058 ;$ Figure $4 \mathrm{~h}$ ). These associations indicated that greater recipient displacement rate coincided with longer, more frequent meals, and less continuous feeding during their meals, whereas the opposite was true for actor displacement rate.

\section{DISCUSSION}

Our results suggested that meal patterns of recently weaned and grouped dairy calves were subject both to previous dietary experience and associated with degree of competition for feed. Previous work has found effects of previous dietary exposure on feed preferences and diet selection (Miller-Cushon et al., 2013b) and effects of previous social contact on meal patterns (Miller-Cushon and DeVries, 2016). Here, we found that previous exposure to hay in any form increased meal frequency after transition to a mixed diet containing starter and hay, suggesting an influence of previous familiarity with feed components on feeding behavior after transition to a mixed diet. Interestingly, however, we found minimal effects of the previous presentation of forage on response to the mixed diet, with no dif- 
Horvath et al.: MEAL PATTERNS OF WEANED CALVES

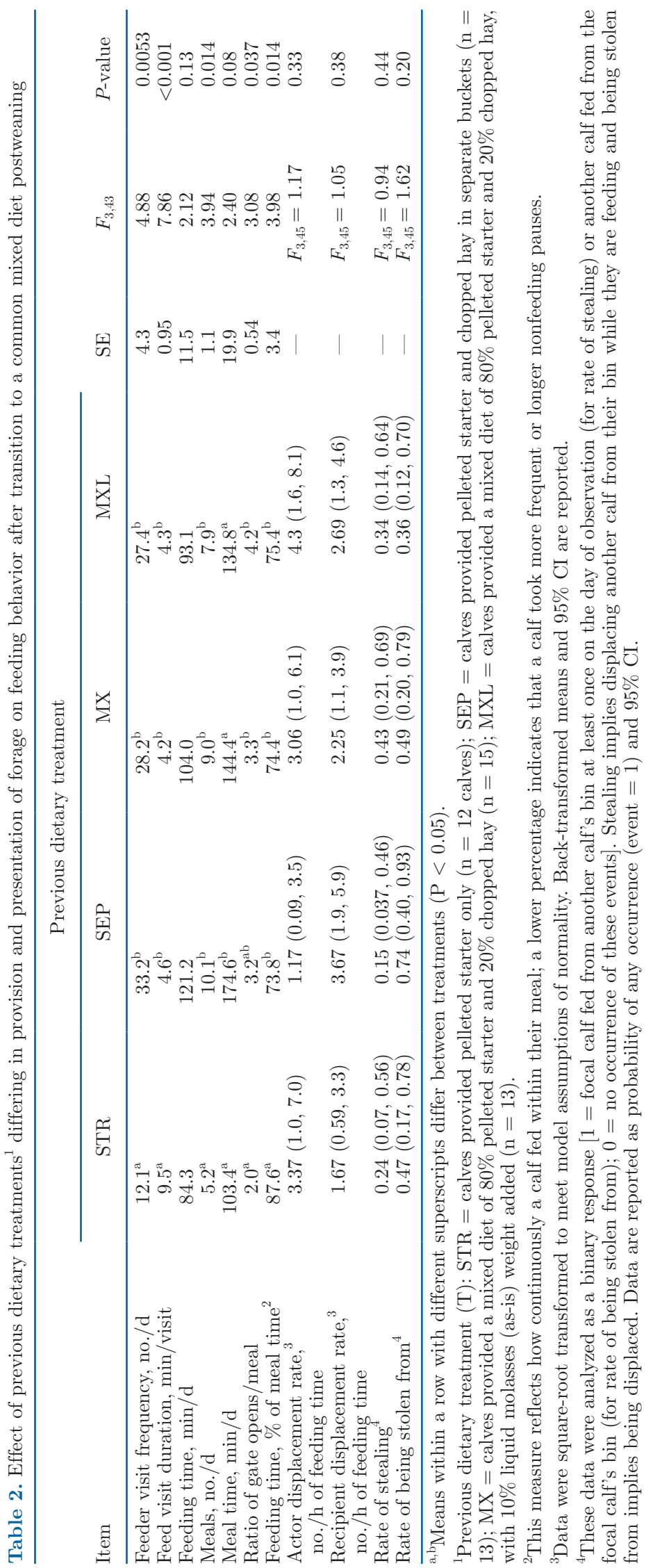



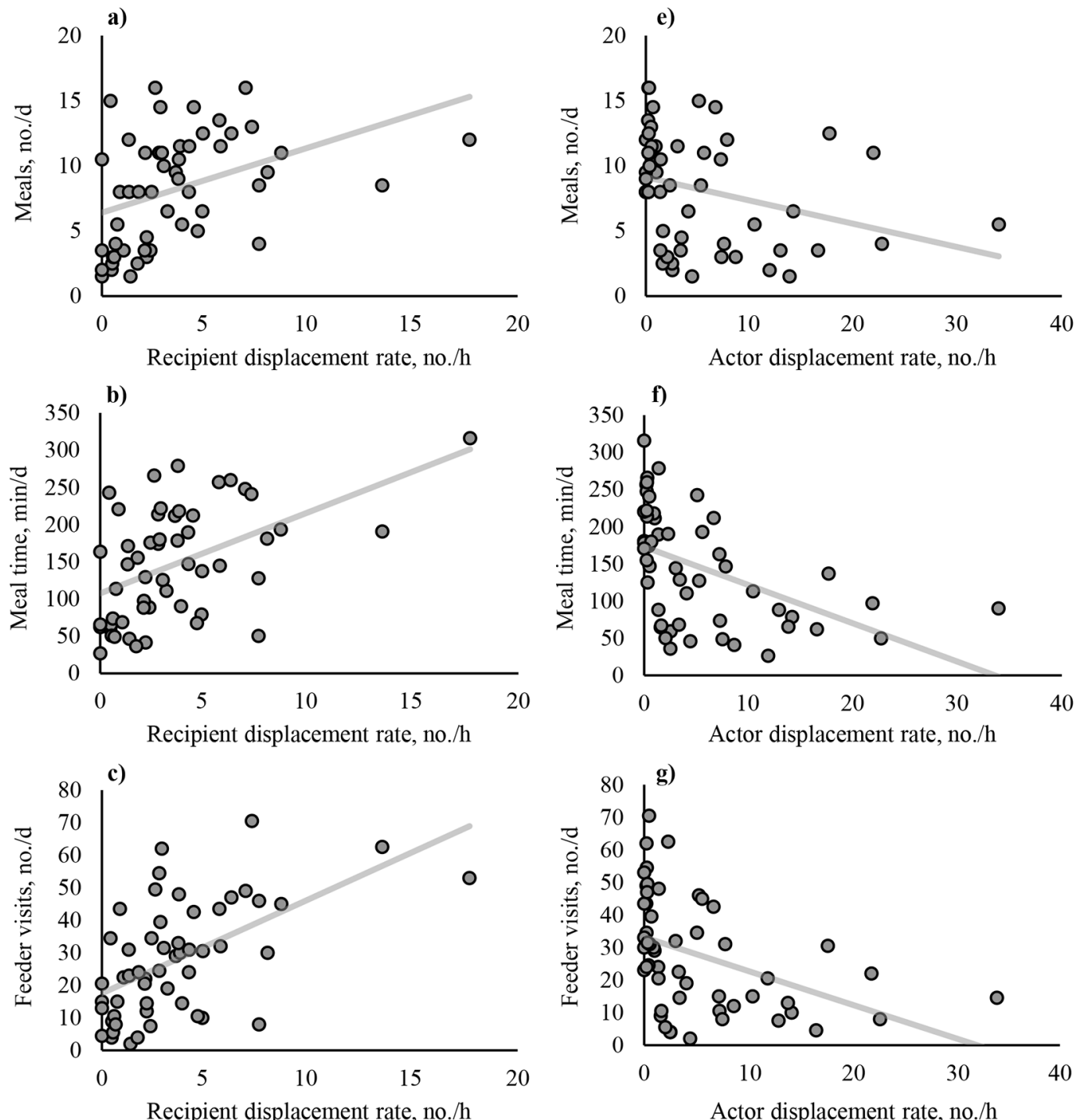

d)
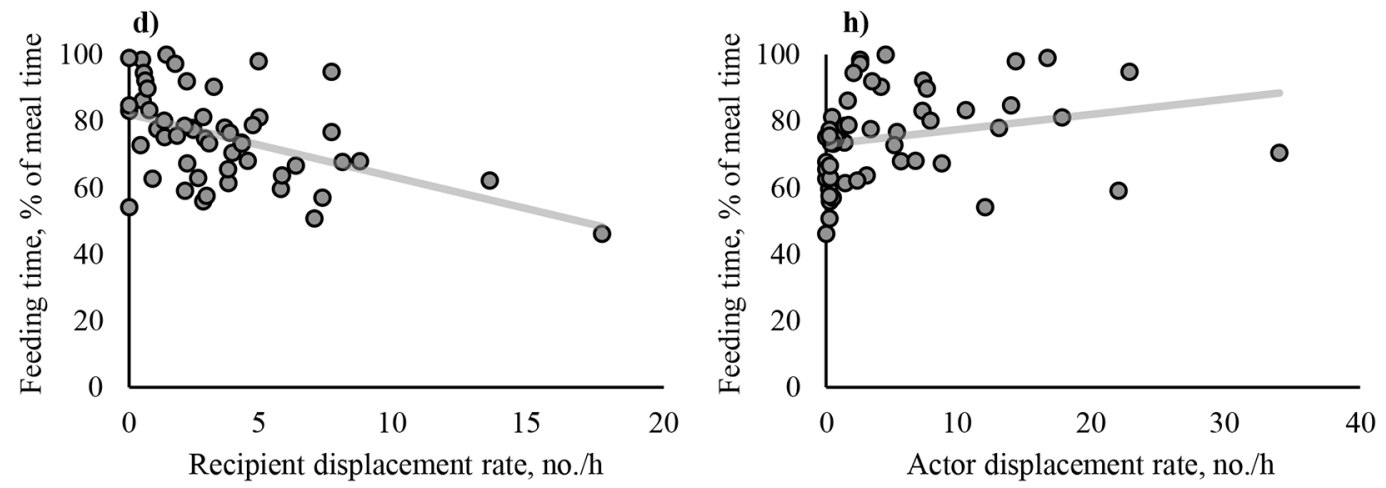

Figure 4. Associations between recipient displacement rate (number of times focal calf was displaced by another calf per hour of feeding time) and daily meal frequency (a), daily duration of meal time (b), frequency of feeder visits (gate opens) per day (c), and feeding time, as a percentage of meal time (d); and between actor displacement (number of times the focal calf displaced another calf per hour of feeding time) and daily meal frequency (e), daily duration of meal time (f), frequency of feeder visits (gate opens) per day ( $\mathrm{g}$ ), and feeding time, as a percentage of meal time $(\mathrm{h})$. 
ferences in meal characteristics between calves previously provided hay separately or as a mixed diet with starter. Although it may be surprising that previous familiarity with the mixed diet offered during the window of observation in this study did not affect meal patterns, compared with exposure to the same ration components offered separately, this result is consistent with previous findings that suggest no effect of forage presentation (with hay provided separately or as a mixture; Miller-Cushon et al., 2013a) on postweaning meal patterns after transition to a common mixed diet. It appears, then, that exposure to feed components, rather than specific method of presentation, may be influential in the development of feeding behavior. In previous work, we found that provision of hay resulted in more frequent, longer meals in preweaning calves (Horvath and Miller-Cushon, 2019). This mirrors the observed differences in meal patterns observed in the present study, suggesting a potential carryover effect of previous meal patterns through a dietary change.

Calves without previous exposure to hay have also avoided that ration component when it is later offered (Miller-Cushon and DeVries, 2011). In the present study, avoidance of unfamiliar feed may explain the observed tendency for reduced feed intake in calves previously provided starter only. However, our measurements of feed intake must be interpreted with caution, given the evidence of feed stealing affecting the majority of calves to the point that we could not exclude data points based on observation of an occurrence of stealing or being stolen from. During our focal observation days, we found that total daily feeding time was not affected by previous dietary experience. We did find that calves previous provided starter only tended to have shorter daily meal times, although this coincided with an increased percentage of feeding time within their meal, suggesting that they actually consumed their ration more steadily.

We found that the extent of competition surrounding feed bins was associated with meal patterns. Feed stealing was rarely reciprocated, with most calves either subject to displacement and stealing from penmates or initiating stealing themselves (as shown for a subset of pens in Figure 2). Actor displacement rate was associated with reduced meal frequency and meal time, and more continuous feeding during meal time, which may imply a greater rate of feed intake. This suggests that increased competition may be detrimental for the calves, or that an increased tendency to steal feed was a symptom of a problem affecting both competitive behavior and feeding time. For example, we could speculate that an avoidance of opening the Calan gate, or dietary aversion and motivation to explore other feed sources, could have possibly resulted in both increased displacements of penmates and reduced feeding time.

Interestingly, calves that were displaced more often had longer meal times and more frequent meals. Increased competitive pressure commonly results in a pattern of larger, less frequent meals [e.g., in adult cattle (Crossley et al., 2017), heifers (DeVries and von Keyserlingk, 2009), and milk-fed calves (Miller-Cushon et al., 2014)], which may be due to an increased cost of accessing the feed. We interpret the present results to suggest that displacements were opportunistic, with calves that spent more time at their feed bin more likely to be targeted by penmates for displacement. Calves that were displaced more often took longer pauses within their meals, which was also observed in adult cows fed by Insentec bins (Crossley et al., 2017), potentially resulting from pauses within meals due to disruption from other calves. In general, these results showed that competition surrounding individual feed bins varied widely between individuals and was associated with meal patterns.

The extent of stealing observed in the present study may not be representative for older animals, and may have been exacerbated by several factors. The young animals observed in the present study may have been able to displace and replace penmates at the feed bin more readily than mature cows, who are able to block the gate more fully. We also focused our observation period on the postweaning transition to social housing, a time point when competition may be increased, as it is established in adult cows that competition increases following regrouping (von Keyserlingk et al., 2008). Calves were also recently introduced to the Calan gate feeding system, which required learning to open the individual gates. Although we did not find a relationship between stealing and ease of learning to open the gate (based on the number of required assists from study personnel), it is possible that hesitancy to open the gates or other subtler differences in ability to access the feed bin contributed to stealing. Our study also involved introducing heifers to the Calan gates at the same point as transition to a novel ration. It is possible that this combination of changes influenced behavior around the Calan gates, although we did not observe effects of previous dietary experience on ability to open the Calan gate or on competition surrounding the gates. We note that a limitation of our study was inconsistent group sizes, due to varying numbers of calves born per week. Although this study was not designed to specifically assess effects of group size on competition and meal patterns of individually-fed heifers, and the group pen was consistently understocked, it is possible that group size affects these behaviors and may contribute to vari- 
ability between groups and calves in the expression of competitive behavior.

We were particularly interested in characterizing competition, including displacements and feed stealing, as this has potential to affect results of studies using individual feed intake control systems. These systems, such as the Calan gates used in the present study and the Insentec Roughage Intake Control system (Instentec BV), are frequently used in research, with the assumption that individuals eat only their own feed. However, as discussed by Ruuska et al. (2014), anecdotal evidence suggests that stealing from individual feeding systems is a frequent problem. Ruuska et al. (2014) describe stealing in cows fed using the Insentec system, with animals either reaching over closed gates or displacing a feeding animal, and noted that it occurred frequently enough to bias results in a study providing different diets where cows preferred one feed over the other. It is likely, however, that occurrences of stealing may go unnoticed in many studies, as a rigorous measurement of competition for feed often relies on video-based observation (Huzzey et al., 2014; Ruuska et al., 2014), and there is infrequent acknowledgment of stealing in the literature. Although analysis of stealing may be possible in systems that record feeding time and access frequency (Huzzey et al., 2014), this information is not recorded by Calan gates, and it necessitated detailed observations from video in the present study.

Where it is reported that cows were excluded due to stealing, in studies using either Insentec bins (Bossen et al., 2009; Bossen and Weisbjerg, 2009) or Calan gates (Jaderborg et al., 2012), the criteria for identifying those animals is not consistently unspecified, and the potential effect on records for animals stolen from is presumably unmeasured. For example, in a report of 48 cows using Calan gates, Jaderborg et al. (2012) stated that 8 animals were identified as outliers resulting from feed stealing, although it is not clear how feed stealing was confirmed as the definitive cause of the outlying data points. Allen et al. (2019) also reported the exclusion of data from 1 animal (within a study of 24 cows fed by Calan gates) due to reduced intake without a change in milk production, which was interpreted to imply that she was stealing from other feed bins. Overall, there is clearly potential for feed stealing in individual feeding systems, but there is limited reliable measurement and reporting of these issues. To our knowledge, the present results are the first to rigorously observe competitive interactions and the extent of feed stealing in heifers fed using the Calan gate individual feeding system. We encourage further work to evaluate the extent of this behavior in different age groups of animals, and the influence of competition on feeding behavior and feed intake.

\section{CONCLUSIONS}

Upon initial social grouping and transition to a common mixed diet, meal patterns of recently weaned dairy calves were influenced by previous dietary experience and associated with competition for access to their assigned feeding bin. Calves without previous exposure to hay had less frequent meals and fed more continuously during their meal time, suggesting a potential carryover of meal patterns from the preweaning period through the dietary transition. We observed frequent displacements of calves from their assigned feeding bin, as well as instances of feed stealing, suggesting that competition may affect behavior of animals fed using feed intake control systems, such as the Calan gates used in this study, to an extent that affects feed intake data. Competition for access to feed appeared to be targeted toward calves that fed for longer, and increased feed stealing coincided with reduced feeding times. Overall, there was considerable individual variability in competitive behavior, which had clear implications for meal patterns. These results highlight the importance of considering both previous dietary experience and social factors when evaluating feeding behavior.

\section{ACKNOWLEDGMENTS}

We thank the staff at the University of Florida Dairy Unit (Hague, FL), our laboratory member Katie Gingerich, and our undergraduate research students (Lisette Coll-Roman, Margaret Fant, Abby Hofmann, Megan McDowd, Giuliana Pezzella, Sam Stella, Tom Wang, and Harrison Warman) for their technical assistance. K. H. was supported by a Matching Assistantship through the Department of Animal Sciences, University of Florida (Gainesville). This research was conducted with support from the Department of Animal Sciences, University of Florida. The authors have not stated any conflicts of interest.

\section{REFERENCES}

Allen, S. C., B. J. Rude, R. H. Bailen, and S. H. Ward. 2019. Reduction of aflatoxin transfer into milk of lactating dairy cows with addition of a dietary clay adsorbent. Bov. Pract. 53:142-148.

Bastian, M., S. Heyman, and M. Jacomy. 2009. Gephi: An open source software for exploring and manipulating networks. International AAAI Conference on Weblogs and Social Media.

Bossen, D., and M. R. Weisbjerg. 2009. Allocation of feed based on individual dairy cow live weight changes: II: Effect on milk production. Livest. Sci. 126:273-285. https://doi.org/10.1016/j.livsci .2009.07.011.

Bossen, D., M. R. Weisbjerg, L. Munksgaard, and S. Højsgaard. 2009. Allocation of feed based on individual dairy cow live weight changes: I: Feed intake and live weight changes during lactation. Livest. Sci. 126:252-272. https://doi.org/10.1016/j.livsci.2009.07.010.

Crossley, R. E., A. Harlander-Matauschek, and T. J. DeVries. 2017. Variability in behavior and production among dairy cows fed un- 
der differing levels of competition. J. Dairy Sci. 100:3825-3838. https://doi.org/10.3168/jds.2016-12108.

DeVries, T. J., and M. A. G. von Keyserlingk. 2009. Competition for feed affects the feeding behavior of growing dairy heifers. J. Dairy Sci. 92:3922-3929. https://doi.org/10.3168/jds.2008-1934.

Engelking, L. E., T. Matsuba, K. Inouchi, T. Sugino, and M. Oba. 2020. Effects of feeding hay and calf starter as a mixture or as separate components to Holstein calves on intake, growth, and blood metabolite and hormone concentrations. J. Dairy Sci. 103:44234434. https://doi.org/10.3168/jds.2019-17676.

Friard, O., and M. Gamba. 2016. BORIS: A free, versatile open-source event-logging software for video/audio coding and live observations. Methods Ecol. Evol. 7:1325-1330.

Horvath, K. C., A. N. Brocious, and E. K. Miller-Cushon. 2021. Effects of forage presentation and addition of molasses-based liquid feed on dairy calf dietary selection and feed sorting of mixed diets. J. Dairy Sci. 104:9052-9062. https://doi.org/10.3168/jds.2021-20422.

Horvath, K. C., and E. K. Miller-Cushon. 2018. Characterizing social behavior, activity, and associations between cognition and behavior upon social grouping of weaned dairy calves. J. Dairy Sci. 101:7287-7296. https://doi.org/10.3168/jds.2018-14545.

Horvath, K. C., and E. K. Miller-Cushon. 2019. Characterizing grooming behavior patterns and the influence of brush access on the behavior of group-housed dairy calves. J. Dairy Sci. 102:3421-3430. https://doi.org/10.3168/jds.2018-15460.

Huzzey, J. M., D. M. Weary, B. Y. F. Tiau, and M. A. G. von Keyserlingk. 2014. Short communication: Automatic detection of social competition using an electronic feeding system. J. Dairy Sci. 97:2953-2958. https://doi.org/10.3168/jds.2013-7434.

Jaderborg, J. P., D. M. Paulus, R. Ginf, F. Diez-Gonzalez, G. I. Crawford, A. DiCostanzo, and J. Drouillard. 2012. DDGS and E. coli in cattle diets: A two-part study. Accessed Jun. 10, 2021. https: //www.auri.org/wp-content/uploads/2012/12/12-12-AIC045-ddgs -ecoli.pdf.

Khan, M. A., D. M. Weary, and M. A. G. von Keyserlingk. 2011. Hay intake improves performance and rumen development of calves fed higher quantities of milk. J. Dairy Sci. 94:3547-3553. https://doi .org/10.3168/jds.2010-3871.

Macdonald, P., and J. Du. 2018. mixdist: Finite Mixture Distribution Models. R package version 0.5-5. https://CRAN.R-project.org/ package $=$ mixdist
Miller-Cushon, E. K., R. Bergeron, K. E. Leslie, G. J. Mason, and T. J. DeVries. 2013a. Effect of feed presentation on feeding patterns of dairy calves. J. Dairy Sci. 96:7260-7268. https://doi.org/ 10.3168/jds.2013-7013.

Miller-Cushon, E. K., R. Bergeron, K. E. Leslie, G. J. Mason, and T. J. DeVries. 2013b. Effect of early exposure to different feed presentations on feed sorting of dairy calves. J. Dairy Sci. 96:4624-4633. https://doi.org/10.3168/jds.2013-6670.

Miller-Cushon, E. K., R. Bergeron, K. E. Leslie, G. J. Mason, and T. J. DeVries. 2014. Competition during the milk-feeding stage influences the development of feeding behavior of pair-housed dairy calves. J. Dairy Sci. 97:6450-6462. https://doi.org/10.3168/jds 2014-8065.

Miller-Cushon, E. K., and T. J. DeVries. 2011. Effect of early feed type exposure on diet-selection behavior of dairy calves. J. Dairy Sci. 94:342-350.

Miller-Cushon, E. K., and T. J. DeVries. 2015. Invited review: Development and expression of dairy calf feeding behaviour. Can. J. Anim. Sci. 95:341-350. https://doi.org/10.4141/cjas-2014-163.

Miller-Cushon, E. K. and T. J. DeVries. 2016. Effect of social housing on the development of feeding behavior and social feeding preferences of dairy calves. J. Dairy Sci. 99:1406-1417. https://doi.org/ $10.3168 /$ jds. $2015-9869$

NRC. 2001. Nutrient Requirements for Dairy Cattle. 7th rev. ed. Natl. Acad. Sci.

Ruuska, S. W., A. Hämäläinen, A. Sairanen, E. Juutinen, L. Tuomisto, M. Järvinen, and J. Mononen. 2014. Can stealing cows distort the results of feeding trials? An experiment for quantification and prevention of stealing feed by dairy cows from roughage intake control feeders. Appl. Anim. Behav. Sci. 159:1-8. https://doi.org/10.1016/ j.applanim.2014.08.001.

von Keyserlingk, M. A. G., D. Olenick, and D. M. Weary. 2008. Acute behavioral effects of regrouping dairy cows. J. Dairy Sci. 91:10111016. https://doi.org/10.3168/jds.2007-0532.

\section{ORCIDS}

K. C. Horvath @ https://orcid.org/0000-0002-5345-6509

A. N. Brocious @ https://orcid.org/0000-0002-5575-4286

E. K. Miller-Cushon (ํ) https://orcid.org/0000-0003-1876-807X 